\section{Tratamiento de lesión periapical con obturación retrógrada e injerto óseo. Reporte de un caso}

\section{Periapical lesion treatment with retrograde filling and bone graft. A case report}

\section{Resumen}

El objetivo de este estudio es presentar un caso en el que tras el fracaso del tratamiento endodóntico se observó una lesión quística en el periápice. El presente caso trata de una paciente femenina de 39 ańos de edad, sin antecedentes médicos relevantes quien asistió a la clínica odontológica SERODU, determinando a la palpación la presencia de una tumefacción de consistencia blanda que estaría afectando la integridad del tejido óseo, sin sintomatología. Las pruebas de vitalidad pulpar resultaron negativas. Al examen radiográfico se observó una sombra radiolúcida compatible con lesión periapical crónica que afecta a las piezas dentales 11 y 12. Por lo que se decidió llevar a cabo la cirugía periapical con apicectomía, sellado apical retrógrado y enucleación de la lesión quística, seguido de regeneración ósea guiada. A los tres meses de control, radiográficamente se encontró una zona radiopaca compatible con regeneración ósea con un halo radiolúcido de separación con el hueso basal original, y a los seis meses, se observó la regeneración ósea completa. La técnica de obturación retrógrada e injerto óseo constituye una técnica de tratamiento efectivo para el sellado del conducto a nivel apical y la regeneración de defectos periapicales de gran tamańo.

Palabras clave: Apicectomía; Endodoncia; Injerto óseo; Quiste radicular; Obturación retrógrada (fuente: DeCS Bireme).

\begin{abstract}
The aim of this study is to present a case in which after the endodontic treatment failed, a cystic lesion was observed in periapical area. The present case concerns a 39 years old female patient without relevant medical history, attended SERODU dental clinic, on clinical examination during the palpation the presence of a soft consistency tumefaction that would be affecting the integrity bone tissue, without symptomatology. The pulp vitality tests turned out to be negative. A radiolucidal shadow compatible with chronic periapical lesion affecting 11 and 12 teeth was observed during the radiographic examination. So it was decided to perform periapical surgery with apicectomy, apical retrograde filling and cystic injury enucleation, followed by guided bone regeneration.
\end{abstract}

\section{Caso Clínico}

María José Jarrín Peñafiel 1,a Jaime Arturo Castro Pallaroso ${ }^{2, b}$, Vicente Mauricio Toscano Viteri ${ }^{3, b}$

1 Universidad Tecnológica Equinoccial, Facultad de Odontología, Quito, Ecuador.

a Odontóloga.

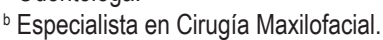

\section{Correspondencia:}

María José Jarrín

Correo electrónico: majp_1995@outlook.com

Calle Bolívar 6-38 y González Suárez, Quito, Ecuador.

\section{Coautores:}

Jaime Arturo Castro Pallaroso

ja.castro@udlanet.ec

Vicente Mauricio Toscano Viter

vicenteodontocirugia@yahoo.com

\section{Editora:}

Marieta Petkova-Gueorguieva

Universidad Nacional Mayor de San Marcos, Perú.

Conflicto de intereses: los autores declaran no tener conflictos de interés.

Fuente de financiamiento: autofinanciado.

\section{Recibido: 10/04/19}

Aceptado: 22/06/19

Publicado: $12 / 09 / 19$

(C) Los autores. Este artículo es publicado por la revista Odontología Sanmarquina de la Facultad de Odontología, Universidad Nacional Mayor de San Marcos. Este es un artículo de acceso abierto, distribuido bajo los términos de la licencia Creative Commons Atribucion - No Comercia_Compartir Igual 4.0 Internacional. (http://creativecommons.org/licenses/by-nc-sa/4.0/) que permite el uso no comercial, distribución y reproducción en cualquier medio, siempre que la obra original sea debidamente citada. 
At three months of control, radiographically a radiopaque area compatible with bone regeneration was found with a radiolucid halo separating with the original basal bone, and at six months, complete bone regeneration was observed. The retrograde filling and bone grafting technique is an effective treatment technique for apical canal sealing and regeneration of large periapical defects.

Keywords: Apicoectomy; Bone graft, Endodontics; Radicular cyst; Retrograde obturation (source: MeSH NLM).

\section{Introducción}

El quiste radicular o periapical, de origen inflamatorio, sigue siendo el quiste odontogénico más frecuentemente descrito ${ }^{1}$. Se forma a partir de restos de Malassez en el ligamento periodontal, como resultado de periodontitis apical posterior a la necrosis pulpar ${ }^{2}$.

Según su tamaño pueden cursar o no con sintomatología facial, aparición de trayectos fistulosos, desplazamiento de dientes adyacentes y afectación de regiones anatómicas cercanas ${ }^{3}$. El quiste radicular odontogénico tiene mayor prevalencia en los dientes del sector anterior del maxilar superior ${ }^{4} \mathrm{y}$ en los del sector posterior de la mandíbula ${ }^{5}$. La localización de la lesión es de especial importancia para evaluar la proximidad que tiene con estructuras orales vecinas como para establecer un correcto plan de tratamiento ${ }^{6}$.

El quiste periapical se diagnostica radiográficamente, se observa un área radiolúcida redondeada u oval, de bordes bien definidos, localizada con más frecuencia en el ápice (raíz) de los dientes ${ }^{7}$.

Cuando la lesión no es tratada suele aumentar de tamańo y producir repetidas infecciones, destruyendo en mayor extensión al hueso afectado y los dientes adyacentes a la infección ${ }^{8}$. Cuando la lesión periapical no se ha recuperado con la endodoncia pueden quedar dos alternativas: la exodoncia del diente y la cirugía apical ${ }^{9}$.

En la actualidad la cirugía periapical se ha convertido en una herramienta importante para preservar aquellas piezas dentales que presentan patología periapical, es el último recurso disponible para resolver los procesos inflamatorios en la zona periapical después del fracaso de un tratamiento de conductos ${ }^{10}$. Este procedimiento consiste en exponer el ápice del diente involucrado, curetaje de los tejidos periapicales, corte del ápice, preparación ultrasónica del ápice y colocación de un material para sellar la cavidad. Idóneamente, este procedimiento debería remover los irritantes del sistema de conductos radiculares y tejidos periapicales, para permitir la regeneración o reparación de los tejidos ${ }^{11}$.

El empleo de injertos óseos como alternativa de reconstrucción de defectos óseos, ya sean congénitos u ocasionados como traumatismos, secuelas oncológicas e infecciosas, tienen como finalidad restablecer la integridad anatómica y funcional de una estructura alterada ${ }^{12}$. Desde hace algún tiempo, se ha demostrado que en los casos con defectos óseos que son tratados con materiales de relleno, se logra una cicatrización del defecto más temprano que en casos en los cuales no se coloca ningún tipo de injerto óseo ${ }^{13}$.

Sin embargo, cuando no existe una adecuada relación corona-raíz el corte del ápice puede llegar a comprometer la estabilidad del diente dentro de su alvéolo, por lo que el conservar la longitud total del órgano dentario es un objetivo a alcanzar ${ }^{14}$. Con la utilización de puntas de ultrasonido se logra preparar una cavidad apical estrecha, larga, de paredes paralelas, sacrificando poco tejido dentario, disminuyendo el riesgo de perforaciones radiculares y facilitando la colocación del material retrógrado, este material de obturación debe tener la capacidad de sellar a las bacterias y sus productos dentro del conducto radicular impidiendo su salida hacia los tejidos periapicales y permitiendo la regeneración de los tejidos ${ }^{15}$.

En el caso reportado, se observó la presencia de una imagen radiolúcida en la región de la pieza dentaria $\mathrm{N}^{\circ} 11 \mathrm{y}$ 12 , que se mantuvo después del tratamiento endodóntico convencional. Razón por la cual se realizó la cirugía periapical con apicectomía, colocación de Agregado de Trióxido Mineral (MTA) y también injerto óseo.

\section{Reporte del caso}

Paciente refiere que hace aproximadamente tres días sintió un edema a nivel del labio superior, lado derecho y observó que el diente había cambiado a una coloración obscura. Refiere también hace 10 años aproximadamente haber sufrido un traumatismo a nivel de las piezas dentales No 11 y 12 producto del cual tuvo movilidad dentaria y cambio de coloración de las piezas dentales, con ausencia de dolor, durante todo este tiempo no ha recibido medicación.

Exámenes complementarios. Radiografía periapical de las piezas dentales No 11 y 12 (Figura 1A).

Diagnóstico. Necrosis pulpar con periodontitis apical crónica más quiste periapical de las piezas dentales No 11 y 12.

Plan de tratamiento. Se planteó realizar tratamiento endodóntico convencional en las piezas dentales No 11 y 12; adicional al día siguiente, se propuso el tratamiento quirúrgico mediante enucleación de la lesión quística, curetaje periapical y apicectomía con retro-obturación de MTA de los órganos involucrados y la colocación de injerto óseo para favorecer la regeneración ósea de la cavidad quirúrgica.

Ejecución - evolución del plan de tratamiento. Se realizó profilaxis a la paciente, se colocó aislamiento absoluto utilizando dique de goma (Dental Dam, Nic- 
Tone, Jalisco, México), se eliminó el material de obturación provisional y se llevó a cabo el acceso endodóntico, utilizando una fresa redonda mediana No5 (SS White, Lakewood, New Jersey); una vez visualizado hidróxido de calcio el conducto se irrigó abundantemente con $\mathrm{NaOCl}$ al 5,25\% para neutralizar el contenido séptico- tóxico. Se abordó corono-apical utilizando limas manuales K-flex \#40, 35,30,25,20 (Dentsply Maillefer, Ballaigues, Suiza) para la preparación de los tercios coronal y medio. Se procedió a la toma de conducto- metría con apoyo de un localizador electrónico (Dentaport-Root-Zx-Morita) y limas manuales K-flex \#20 (Dentsply Maillefer, Ballaigues,Suiza).

La preparación biomecánica fue realizada con técnica manual hasta lograr un diámetro apical \#45 e irrigación manual utilizando $\mathrm{NaOCl} 5,25 \%$. En esta primera cita se decidió colocar medicación intraconducto de (Ultracal, Ultradent Products INC, Monterrey, México), una obturación temporal a base de óxido de zinc (Provisit, Casa Idea, San Luis Potosí, México).

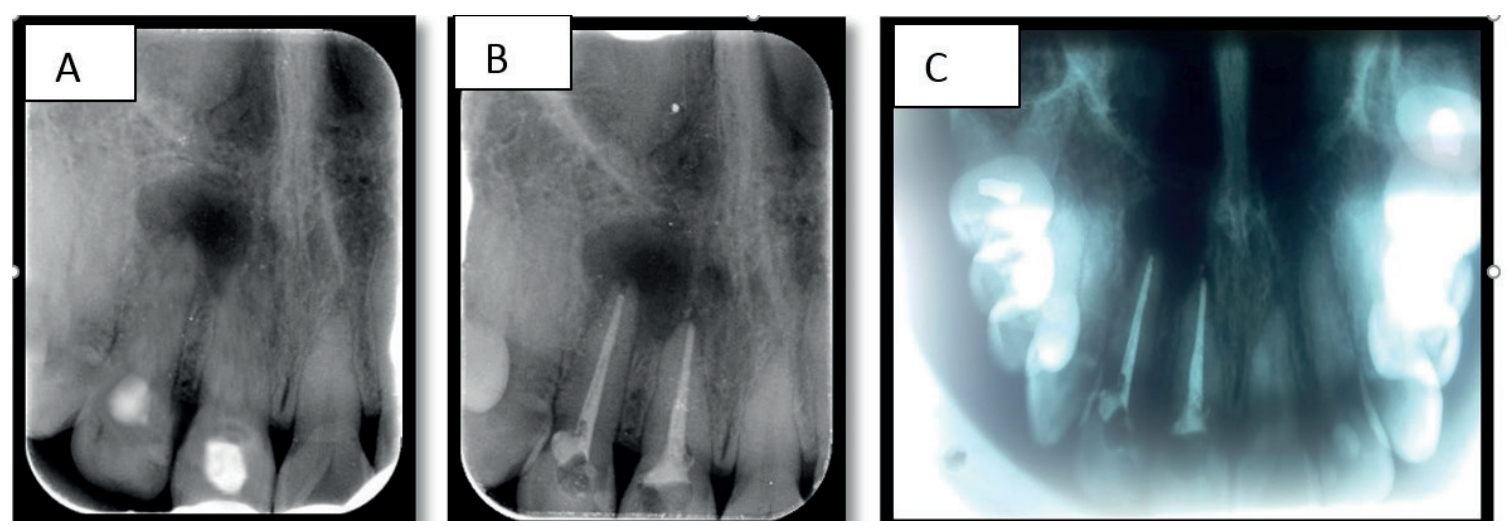

Figura 1. A. Radiografía periapical Inicial de la Pieza № 11 y 12. B. Radiografía periapical de obturación. C. Radiografía oclusal superior de obturación

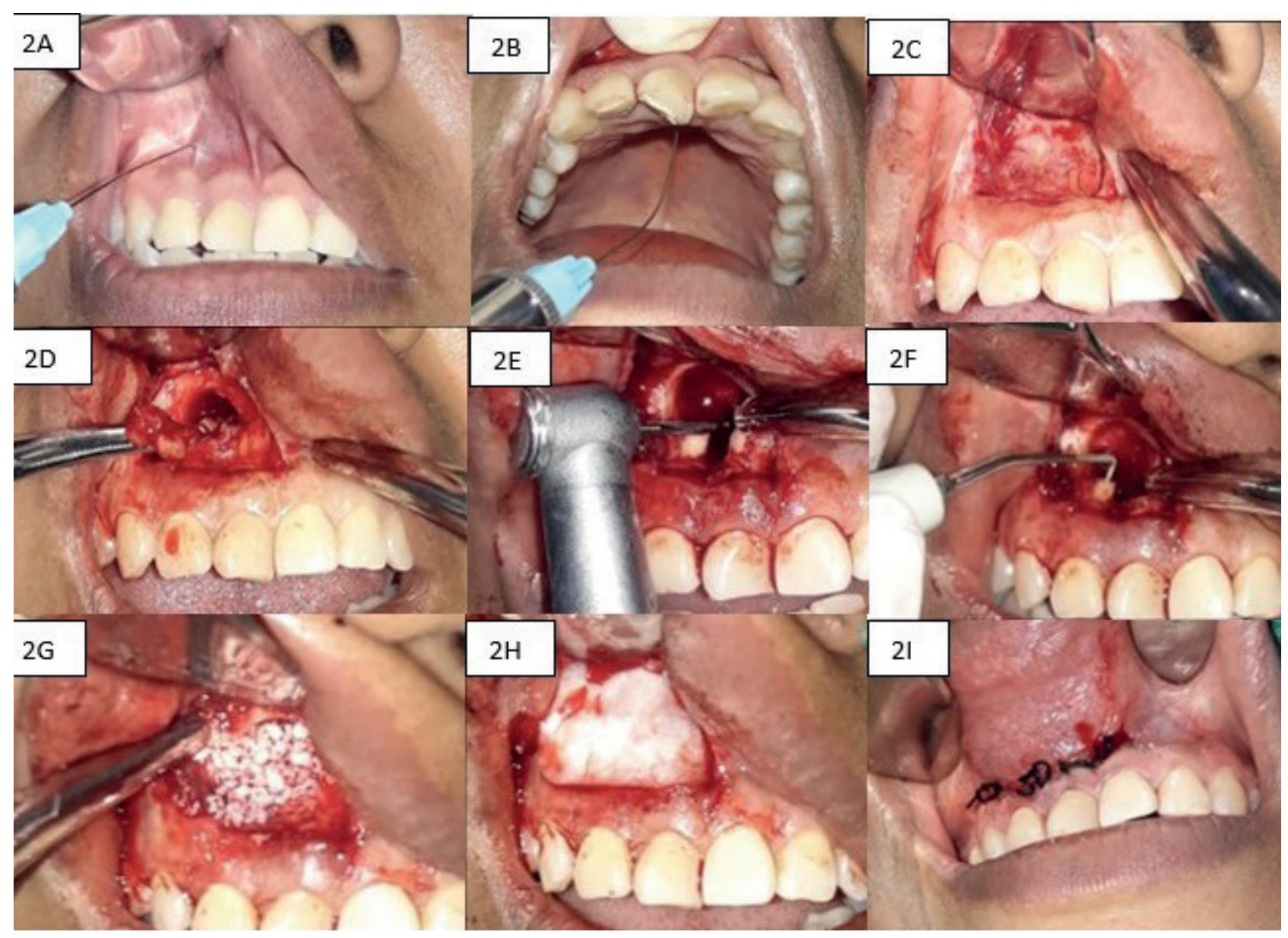

Figura 2. A. Bloqueo anestésico del nervio alveolar anterior. B. Bloqueo anestésico del nervio nasopalatino. C. Levantamiento de colgajo. D. Enucleación de la lesión periapical. E. Apicectomía. F. Obturación retrógrada. G. Colocación de injerto óseo. H. Colocación de membrana reabsorbible. I. Reposicionamiento de colgajo y sutura 

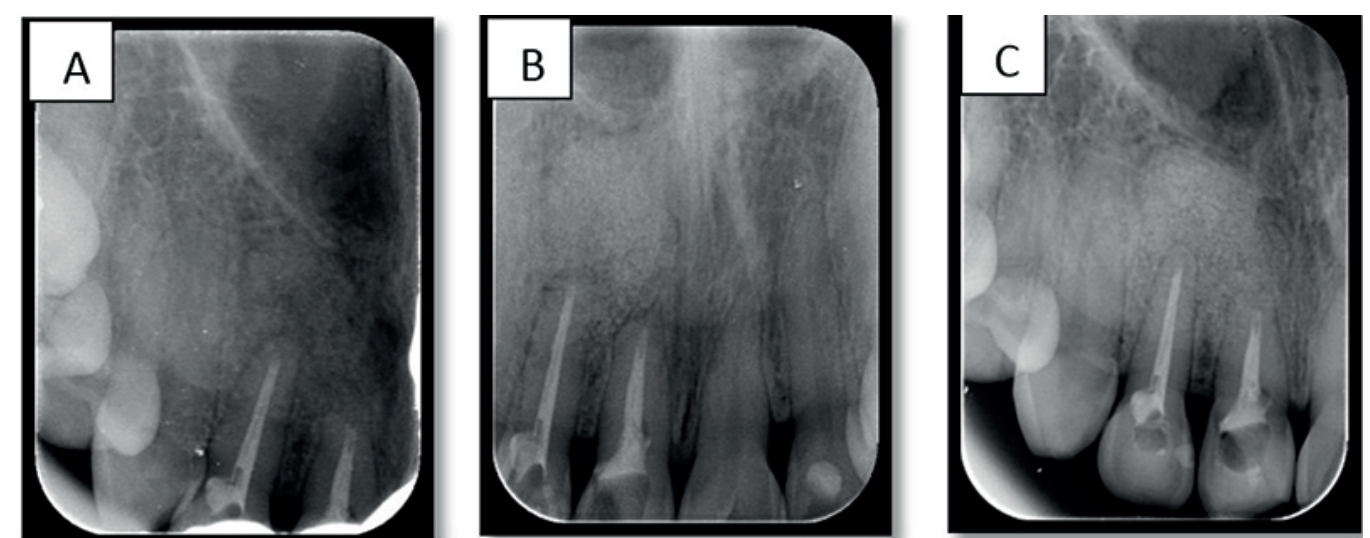

Figura 3. A. Radiografía postoperatoria de la pieza № 11 y 12. B. Radiografía postoperatoria a los 3 meses. C. Radiografía postoperatoria a los 6 meses

En una segunda cita, se eliminó la obturación temporal con fresa redonda \#5 de alta velocidad y la medicación intraconducto mediante irrigación manual con $\mathrm{NaOCl}$ $5,25 \%$, el conducto de la pieza No11 fue finalmente instrumentado a una lima apical \#60 y el conducto de la pieza No12 a una lima apical \#55. Se utilizó EDTA 17\% (Ultradent Products INC, Monterrey, México) durante 1 minuto como acondicionamiento final del conducto. Se llevó a cabo el secado completo de los conductos utilizando conos de papel \#35 y se procedió a realizar la conometría y finalmente se decidió llevar a cabo la obturación de los conductos mediante técnica de compactación lateral con un cono maestro de gutapercha estandarizada \#60 en la pieza No 11 y un cono \#55 en la pieza No 12 (Hygenic, ColteneEndo, Altstatten, Suiza) y conos accesorios B (Hygenic, ColteneEndo, Altstatten, Suiza) utilizando cemento sellador a base de hidróxido de calcio (Sealapex, SybronEndo, Orange, California, E.U.A.). Finalmente se colocó una obturación de ionómero de vidrio (Fuji 9, GC Corporation, Tokio, Japón) (Figura 1B y 1C).

En la siguiente cita se decidió llevar a cabo la cirugía apical con apicectomía para la eliminación directa de la lesión. Se procedió a la colocación de la mesa quirúrgica, se realizó la asepsia y antisepsia de la zona quirúrgica. Se colocó los campos quirúrgicos a la paciente. Se inició con el bloqueo anestésico del nervio alveolar superior anterior (Figura 2A), así como el nervio nasopalatino (Figura 2B), se utilizó anestesia con lidocaína al 2\% con epinefrina 1:100 000 .

Para la incisión se utilizó mango de bisturí o 3 y hoja $15 c$, fue efectuada profundizando hasta la cortical ósea e incidiendo el periostio. Se continuó con la elevación del colgajo de espesor total con la ayuda de la legra. El manejo del colgajo se realizó mediante un separador de Minnesota, aplicando ligera presión en el hueso para evitar la isquemia del colgajo (Figura 2C). Se realizó la osteotomía con fresa quirúrgica redonda $\# 708$ de baja velocidad.

Una vez expuestas las raíces de los órganos a tratar, se realizó la completa enucleación de la lesión, con la cureta de Lucas se eliminó el tejido de granulación (Figura 2D). Se procedió a la resección de los tres milímetros apicales del diente No 11 con instrumentos rotatorios utilizando una fresa Zecrya (Figura 2E). Se retro-prepararon con punta angulada ultrasónica seguida de la retro-obturación apical con MTA) con una fresa redonda 1012 (Angelus, Londrina, Brasil) (Figura 2F). Se llevó a cabo el lavado de la cavidad remanente con abundante solución fisiológica para eliminar restos de tejido, cálculo dental o cemento y proporcionar una zona libre de contaminación ideal para la colocación del injerto Geistlich Bio-Oss ${ }^{\oplus} /$ Geistlich Bio-Oss Pen ${ }^{\oplus}$ (Figura $2 \mathrm{H})$, y por último se cubrió con una membrana de colágeno Geistlich Bio-Gide al injerto óseo (Figura 2G).

Terminado el procedimiento quirúrgico, se procedió a reposicionar el colgajo el cual fue suturado mediante puntos simples a lo largo de la incisión con material de seda, trenzada, no reabsorbible (Figura 2I). Una vez realizada la sutura, se colocó una gasa estéril y se le pidió al paciente que ocluyera para favorecer la formación del coágulo. Se le entregó por escrito receta médica e indicaciones postoperatorias. Se tomó una radiografía periapical postoperatoria (Figura 3A).

Seguimiento del tratamiento ejecutado. Las suturas fueron retiradas a los 15 días de realizado el procedimiento quirúrgico. A la exploración clínica se observó inflamación papilar leve sin signos de infección y evolución satisfactoria del proceso de cicatrización. Se le indicó al paciente retomar su dieta normal y la periodicidad en que deberá presentarse a consulta para evaluar los resultados obtenidos, a los 3 y 6 meses.

Evaluación Postoperatoria a los 3 meses. El paciente no refirió sintomatología. Clínicamente no se observaron signos de recidiva de la lesión. Se tomó radiografía de la zona tratada en la cual se observa una zona radiopaca compatible con regeneración ósea con un halo radiolúcido de separación con el hueso basal original (Figura 3B).

Evaluación Postoperatoria a los 6 meses. A los seis meses de control, se observó la regeneración ósea mediante control radiográfico (Figura 3C). El hueso utilizado se colocó con el fin de rellenar el espacio dejado por la lesión periapical y con el fin de proteger el ápice de la raíz y promover una mejor y más rápida cicatrización ósea. 


\section{Discusión}

Mediante el tratamiento propuesto cirugía apical más apicectomía conjugado con injerto óseo fue posible preservar los dientes $\mathrm{N}^{\circ} 11$ y 12 en boca del paciente eliminado el quiste que afectaba a los dientes $\mathrm{N}^{\circ} 11$ y 12 . Según Bramante y Herbert en el año 2000, en situaciones en las que el tratamiento endodóntico es realizado como único procedimiento es muy difícil conseguir la resolución de un problema asociado a la presencia de un quiste ${ }^{16}$. De ahí por qué fue seleccionada la ejecución de esta manobra quirúrgica, a esto se suma el hecho de que la persistencia de la lesión radiolúcida en la zona apical es muy frecuente, incluso después del tratamiento y retratamiento endodóntico según reportado por diversos autores ${ }^{17}$.

La radio transparencia relacionada con un quiste periapical siempre es redonda u ovoide con un halo radiopaco contigua a la lámina dura del diente afectado ${ }^{18}$, varían de $5 \mathrm{~mm}$ a varios centímetros de diámetro ${ }^{19}$. En quistes que han permanecido largo tiempo se puede detectar resorción de la raíz del diente afectado y ocasionalmente de dientes adyacentes ${ }^{20}$. Se ha reportado desde un $37 \%$ hasta un $97 \%$ de curación completa después de la cirugía apical y regeneración ósea guiada ${ }^{21}$. La presencia o ausencia de signos y síntomas se utilizan para evaluar el resultado del tratamiento como exitoso ${ }^{22}$.

La relación entre el pronóstico y el tipo de diente no está muy claro todavía, muchos autores coinciden en que los incisivos y los caninos tienen mayor tasa de éxito ${ }^{23,24}$, lo que puede explicarse porque los sectores anteriores son más accesibles y permiten una excelente vista del campo operatorio, favoreciéndose obtener un mejor sellado apical. Barone et al. ${ }^{25}$ encontraron que lesiones menores o igual a $10 \mathrm{~mm}$ tenían una tasa de éxito.

La cirugía apical con apicectomía se puede realizar el mismo día que se realiza la obturación convencional o 72 horas después ${ }^{26}$.

Los lechos quirúrgicos dejados por las lesiones quísticas pueden ser lavados con suero fisiológico, agua oxigenada o clorhexidina al $2 \%{ }^{27}$.

Se lleva a cabo el corte de la raíz en ángulo de 45 grados, lo que favorece la visión clara del conducto radicular y material de obturación, técnica aplicada desde 1996 por el Dr. Del Río ${ }^{28}$. Se realiza una resección apical de $3 \mathrm{~mm}$ de longitud respecto al eje axial de la raíz recomendado por Wälivaara et al. en sus estudios ${ }^{29}$. Bateman $\mathrm{G}$ et al. ${ }^{30}$ en el año 2010 demostraron que la cirugía periapical y obturación retrógrada mediante técnicas de ultrasonidos son capaces de realizar preparaciones apicales de mayor calidad que los instrumentos rotatorios, es por eso que se utilizó ultrasonido.

Como desventajas, se ha comentado que la vibración originada por los ultrasonidos en la obturación retrógrada puede ocasionar microfracturas dentarias por lo que se recomienda la cauterización de cono desde el ápex ${ }^{31}$.

En la obturación retrógrada se utilizó MTA debido a que Tang et al. ${ }^{32}$ en el año 1997 mediante sus estudios informaron que gracias a la naturaleza hidrofílica y la expansión del MTA en un medio húmedo; se obtiene un mayor sellado, evitando de este modo la microfiltración y la infiltración de las endotoxinas bacterianas.

Las técnicas de regeneración ósea guiada en combinación con injertos óseos pueden ser utilizadas para la reconstrucción de grandes lesiones periapicales con una elevada tasa de éxito ${ }^{33}$. El uso de membranas barrera impide la penetración y el contacto del tejido conectivo con las paredes del defecto óseo, permitiendo la recolonización del mismo por células propias del tejido óseo, a la vez que protege y estabiliza el coágulo sanguíneo subyacente que se ha formado ${ }^{34}$.

La técnica de obturación retrógrada e injerto óseo constituye una técnica de tratamiento efectivo para el sellado del conducto a nivel apical y la regeneración de defectos periapicales de gran tamaño.

En las lesiones endoperiodontales es necesario realizar tanto el tratamiento endodóntico, como el periodontal, siendo el tratamiento endodóntico habitualmente el que se realiza primero.

\section{Referencias bibliográficas}

1. Regezi JA, Sciubba JJ. Patología bucal: correlaciones clínico patológicas. 3ra. ed. Philadelphia: McGraw-Hill Interamericana; 2010.

2. Ricucci D, Siqueira JF. Recurrent apical periodontitis and late endodontic treatment failure related to coronal leakage: a case report. J Endod. 2011;37(8):1171-5

3. Kruse C, Spin-Neto R, Christiansen R, Wenzel A, Kirkevang LL. Periapical Bone Healing after Apicectomy with and without Retrograde Root Filling with Mineral Trioxide Aggregate: A 6-year Follow-up of a Randomized Controlled Trial. J Endod. 2016;42(4):533-7.

4. Serrano-Giménez M, Sánchez-Torres A, Gay-Escoda C. Prognostic factors on periapical surgery: A systematic review. Med Oral Patol Oral Cir Bucal. 2015;20(6):715-22.

5. Song M, Jung IY, Lee SJ, Lee CY, Kim E. Prognostic factors for clinical outcomes in endodontic microsurgery: a retrospective study. J Endod. 2011;37(7):927-33.

6. Bilodeau EA, Collins BM. Odontogenic Cysts and Neoplasms. Surg Pathol Clin. 2017;10(1):177-222.

7. Öğütlü F, Karaca İ. Clinical and Radiographic Outcomes of Apical Surgery: A Clinical Study. J Maxillofac Oral Surg. [Internet]. 2018 [cited 2018 Aug 15];17(1):7583. Disponible en: https://www.ncbi.nlm.nih.gov/ pubmed/29382999

8. Kreisler M, Gockel R, Aubell-Falkenberg S, Kreisler T, Weihe C, Filippi A, Kühl S et al. Clinical outcome in periradicular surgery: effect of patient- and tooth-related factors--a multicenter study. Quintessence. [Internet]. 2013 [consultado 9 agosto 2018];44(1):5360. Disponible en: https:/www.ncbi.nlm.nih.gov/ pubmed/23444162.

9. Kourkouta S, Bailey GC. Periradicular regenerative surgery in a maxillary central incisor: 7-year results including cone-beam computed tomography. J Endod. 2014;40(7):1013-9. 
10. Von Arx T, Peñarrocha M, Jensen S. Prognostic factors in apical surgery with root-end filling: a meta-analysis. J Endod. 2010;36(6):957-73.

11. Kattimani VS, Chakravarthi SP, Neelima Devi KN, Sridhar MS, Prasad LK. Comparative evaluation of bovine derived hydroxyapatite and synthetic hydroxyapatite graft in bone regeneration of human maxillary cystic defects: a clinico-radiological study. Indian J Dent Res. [Internet]. 2014[consultado 9 agosto 2018];25(5):594601. Disponible en: https://www.ncbi.nlm.nih.gov/ pubmed/25511058.

12. Elgali I, Omar O, Dahlin C, Thomsen P. Guided bone regeneration: materials and biological mechanisms revisited. Eur J Oral Sci. 2017;125(5):315-337.

13. Tsesis I, Rosen E, Tamse A, Taschieri S, Del Fabbro M. Effect of guided tissue regeneration on the outcome of surgical endodontic treatment: A systematic review and meta-analysis. J Endod 2011;37(8):1039-45.

14. Von Arx T, Jensen SS, Hänni S, Friedman S. Five-year longitudinal assessment of the prognosis of apical microsurgery. J Endod. 2012;38(5):70-79.

15. Pop I. Oral surgery: part 2. Endodontic surgery. $\mathrm{Br}$ Dent J. [Internet].2013 [consultado 15 agosto 2018];215(6):279-286. Disponible en: https://www. ncbi.nlm.nih.gov/pubmed/24072296.

16. Bramante CM, Berbet A. Cirugía Paraendodóntica.São Paulo:Santos;2000.

17. Alsulaimani RS. Single-visit endodontic treatment of mature teeth with chronic apical abscesses using mineral trioxide aggregate cement: a randomized clinical trial. BMC Oral Health. 2016;16(1):78.

18. Von Arx T, Janner SF, Jensen SS, Bornstein MM. The resection angle in apical surgery: a CBCT assessment. Clin Oral Investig. 2016;20(8):75-82.

19. Tsesis I, Elbahary S, Venezia NB, Rosen E. Bacterial colonization in the apical part of extracted human teeth following root-end resection and filling: a confocal laser scanning microscopy study. Clin Oral Investig. [Internet].2018; [consultado 15 agosto 2018]; 22(1):267274. Available in: https://www.ncbi.nlm.nih.gov/ pubmed/28349219.

20. Deshmukh J, Shrivastava R, Bharath KP, Mallikarjuna R. Giant radicular cyst of the maxilla. BMJ Case Rep. 2014.

21. Jang Y, Hong HT, Roh BD, Chun HJ. Influence of apical root resection on the biomechanical response of a single-rooted tooth: a 3-dimensional finite element analysis. J Endod. 2014;40(9):1489-93.

22. Riis A, Taschieri S, Del Fabbro M, Kvist T. Tooth Survival after Surgical or Nonsurgical Endodontic Retreat- ment: Long-term Follow-up of a Randomized Clinical Trial. J Endod. 2018;44(10):1480-1486.

23. Chércoles-Ruiz A, Sánchez-Torres A, Gay-Escoda C. Endodontics, Endodontic Retreatment, and Apical Surgery Versus Tooth Extraction and Implant Placement: A Systematic Review. J Endod. 2017;43(5):679-686.

24. Ricucci D, Siqueira JF Jr. Recurrent apical periodontitis and late endodontic treatment failure related to coronal leakage: a case report. J Endod. 2011;37(8):1171-5.

25. Barone C, Dao TT, Basrani BB, Wang N, Friedman S. Treatment outcome in endodontics: the Toronto study--phases 3, 4, and 5: apical surgery. J Endod. 2010;36(1):28-3):76-81.

26. Bernabé PF, Gomes-Filho JE, Cintra LT, Moretto MJ, Lodi CS, Nery MJ et al. Histologic evaluation of the use of membrane, bone graft, and MTA in apical surgery. Oral Surg Oral Med Oral Pathol Oral Radiol Endod. 2010;109(2):309-14.

27. Tawil PZ, Saraiya VM, Galicia JC, Duggan DJ. Periapical microsurgery: The effect of root dentinal defects on short and long term outcome. J Endod. 2015;41(1):22-27.

28. Von Arx T, Peñarrocha M, Jensen S. Prognostic factors in apical surgery with root-end filling: a meta-analysis. J Endod. 2010;36(6):957-973.

29. Wälivaara DA, Abrahamsson P, Sämfors KA, Isaksson $S$. Periapical surgery using ultrasonic preparation and thermoplasticized gutta-percha with $\mathrm{AH}$ Plus sealer or IRM as retrograde root-end fillings in 160 consecutive teeth: a prospective randomized clinical study. Oral Surg Oral Med Oral Pathol Oral Radiol Endod. 2009;108(5):784-9.

30. Bateman G, Coomaraswamy K, Saha S. Mineral trioxide aggregate use in failing endodontic treatment--a case report. Dent Update. 2010;37(1):33-5,38-9.

31. Liu Z, Zhang D, Li Q, Xu Q. Evaluation of rootend preparation with a new ultrasonic tip. J Endod. 2013;39(6):820-3.

32. Tang HM, Torabinejad M, Kettering JD. Leakage evaluation of root end filling materials using endotoxin. J Endod. 2002;28(1):5-7.

33. Taschieri S, Rosano G, Weinstein T, Bortolin M, Del Fabbro M. Treatment ofthrough-and-through bone lesion using autologous growth factors and xenogeneic bone graft: a case report. Oral Maxillofac Surg. 2012;16(1):57-64.

34. Irokawa D, Takeuchi T, Noda K, Goto H, Egawa M, Tomita $S$ et al. Clinical outcome of periodontal regenerative therapy using collagen membrane and deproteinized bovine bone mineral: a 2.5-year follow-up study. BMC Res Notes. 2017;10(1):102. 